\title{
Roles of Multidrug Transporters of MFS in Plant Stress Responses
}

\author{
Hua Peng, Sufen Han, Mao Luo, Jian Gao, Xuan Liu, and Maojun Zhao
}

\begin{abstract}
Multidrug transporters are a class of secondary transporters and important transmembrane transporters which belong to facilitator superfamily (MFS). Multidrug transporters play critical roles in plant defense against various toxin stresses by exporting toxin outside the cell to reduce the accumulation of toxin compounds in body. In this article, structural features and mechanisms of MFS multidrug transporters responsed to plant stresses are described in details, such as defending against the infection of pathogen, exporting toxic compounds and transporting the heavy metal and so on. And then, the molecular modulation mechanism of plants MFS multidrug transporters is preliminarily discussed and the important roles of these transporters are defineds responsed to sresses in plants.
\end{abstract}

Index Terms-MFS, Multidrug Transporters, Plant.

\section{INTRODUCTION}

Plant growth and development are frequently subjected to various environmental stresses which has been the critical limiting factors for plant growth, such as biotic and abiotic stresses. It is revealed that many transporter families of plants participate in responding to pesticides, toxic chemicals and infection of pathogens. These transporters are divided into five characterized families: the ATP binding cassette $(\mathrm{ABC})$, multidrug and toxic compound exporters (MATE), small multidrug resistance (SMR), resistance-nodulation-division proteins (RND), and the major facilitator superfamily (MFS) [1]. Multidrug transporters are a class of membrane transport proteins in MFS, which acted as drug $/ \mathrm{H}^{+}$transporters to reduce the accumulation of toxin in the body by expelling toxic compounds from the cell energized by proton motive force. Presently the mechanism of transmembrane transport of MFS multidrug transporters has become a research topic in the field of plant stress biology and structural biology. The structural characteristics and the important biological functions of MFS multidrug transporters is discussed in this article, such as resisting the intrusion of pathogens, expelling the multiple drugs from the cell and transporting the heavy metal to outside the cell or the region of lower concentration of plant cell in exchange for protons, It is revealed that MFS multidrug transporters plays the delicate roles in the regulatory network of responding to the stress.

Manuscript received July 11, 2011; revised July 23, 2011.

H.Peng, Sichuan Agricultural Management College,Chengdu 610071, China,16828333@qq.com.

S.Han , Life and Science Department, Sichuan Agricultural University, Ya'an 625014, China, han851014@163.com

M.Luo, Drug Discovery Research Center,Luzhou Medical College, Luzhou646000, China.luomao20050908@yahoo.cn.

M.Zhao (Corresponding author), Life and Science Department, Sichuan Agricultural University, Ya'an 625014, China, zmjun01@yahoo.com.cn.

\section{Structure Characteristics OF MFS MUltidRUG TRANSPORTERS}

Major facilitator superfamily (MFS), also called the uniporter-symporter-antiporter superfamily, which belong to the largest and widespread superfamily of secondary active transportersand mediate the translocation of small molecules through chemosmotic ionic gradients[1]. So far, the MFS contains 58 distinct families[3], and about 15,000 sequenced members are identified [4-8].Given the lower sequence similarity between identified members, all the members share the similar protein folding structure and transportation mechanism at the level of the superfamily [9].

Multidrug transporters as a subfamily of membrane transporters of major facilitator superfamily, consist of DHA1 and DHA2, both of which belong to drug: $\mathrm{H}^{+}$ antiport family. Almost all MFS proteins possess a uniform topology of 12 or 14 transmembrane $\alpha$-helices (TMs) connected by large cytoplasmatic hydrophilic loops, with both their N- and C- termini located in the cytoplasm [4-5, 10-11]. 12-TMs topologies consist of C-terminal TM1-TM6 and N-terminal TM7-TM12 and two 6-TMs units whose arrangement are resemble, connected by a hydrophilic loop between TMs 6 and 7. In the 14-TMs members, the extra two helices probably emerged via insertion of the central cytoplasmic loop into the membrane, speculation has also been presented these extra two helices arose by additional acquisition of 2 TMs at the C-terminal domain of the protein. Research shows that N-terminal TM1-TM6 exhibits weaker sequence homology than C-terminal TM7-TM12, suggesting that 12-TMs transporters evolved from a duplication or fusion event of a gene encoding a protein with $6 \mathrm{TMs}[3]$. Further analysis indicated that highly conserved sequences made of 13 amino acids between TM2-TM3 loop and relatively conserved sequences of cytoplasmic TM8-TM9 loop have been identified as the typical characteristics of MFS family [4].

It is found that multidrug transporter EmrD belongs to the 12-TMs antiporter DHA1 family shared same high-resolution three-dimensional (3D) structures with LacY (Escbericbia coli lactose $/ \mathrm{H}^{+}$permease)[3], GlpT (sn-glycerol-3-phosphate: phosphate), and speculated that most all of MFS members likely share similar structure patterns. EmrD shows a more compact, occluded conformation of twelve membrane-spanning $\alpha$-helices forming two six-six bundles, which looks like a hydrophobic cavity within the plane of the bilayer. The external $\alpha$-helices (TM-3,6,9,12) share a similar configuration to the counterparts of $\mathrm{LacY}$ and GlpT, but the internal $\alpha$-helices are different from their equivalents and form a internal cavity, which probable participate in drug transportation, even there is no direct evidence to show the 
connection of drugs in this cavity and exportation from the cavity. However, studies of MdfA and LmrP mutation show that residues (hydrophobic residues, aromatic residues and some polar residues) contribute to substrate recognition and translocation, suggesting this interal cavity is probably a hydrophobic channel for substrate translocation. There is no exact evidence of 14-TM transpoters whether they possess such folding mechanism relative to $12-\mathrm{TM}$ transpoters, new research shows that the interacted residues of QacA 14-TMs located in the interactional helices while drawing the floding helices.

Conservative and variable regions of amino acid sequences of bacteria and plant multidrug transporters from major facilitator superfamily (MFS) were compared using MEGA4, two differences of amino acid sequences between plant and bacteria multidrug transporters are found. First, a distinctly different cytoplasmic loop is located between TM8 and TM9 in plant multidrug transporters of major facilitator superfamily, normally following the pattern $x-[R K]-x(2)-G-P-[I V]-x(3)-R-x$, nevertheless, the same cytoplasmic loop of bacteria have the motif
[GD]-[RK]-x(5)-G-x(2). It is speculated that the difference of TM8-TM9 cytoplasmic loop in plant and bacteria might be associated with recognition of substrates[12]. Since substrate translocation is thought to operate via proton motive force, and structure of TM8-TM9 cytoplasmic loop in plant is distinctly different from their homologies, there may be notably different range of the substrates of plant MFS transporters. The TM8-TM9 loop with aspartate in position one mediated aromatic acid transport [13], and the net with positive charge $(+3)$ of Zm-Mfs1 loop TM8-TM9 propably expel positively charged substrates. Second, the position of His residue and His-Asp residues are different in plant and bacteria. In bacteria, conserved His-Asp residues located in TM8-TM9 loop are thought to participate in proton-mediated substrate antiport. However, His residue is membrane-located in TM7 and next to Asp residue in the plant genes; His-Asp located in TM7 might play the same role in determining the substrate antiport. So, it is speculated that changed position of His-Asp which conserved and adjacently located in TM7 of plant transporters probably shift the transmembrane position of substrates [12].

TABLE 1. SEQUENCES COMPARSION OF CONSERVATIVE AND VARIABLE REGIONS OF PLANT AND BACTERIA MFS TRANSPORTERS

\begin{tabular}{|c|c|c|c|c|c|}
\hline gene., & accession. & $\begin{array}{c}\text { TMI2-3 loop.- } \\
\text { MFS.. }\end{array}$ & $\begin{array}{c}\text { TM15.r } \\
\text { Antiporter. }\end{array}$ & $\begin{array}{l}\text { TMM7.7 } \\
\text { His-Asap.r }\end{array}$ & $\begin{array}{c}\text { TMr8-9 loop.r } \\
\text { Substrate. }\end{array}$ \\
\hline 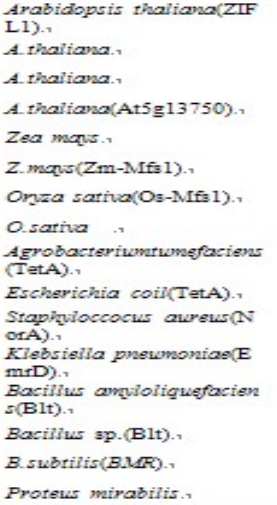 & 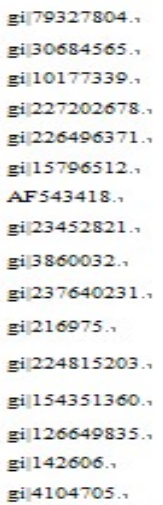 & 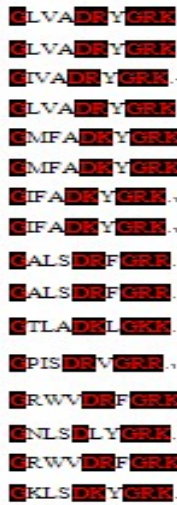 & 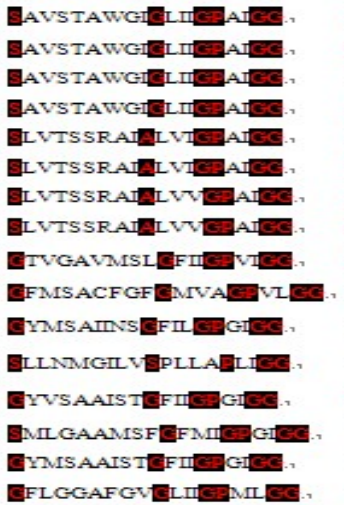 & 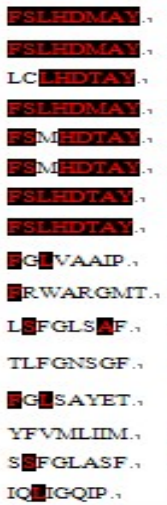 & 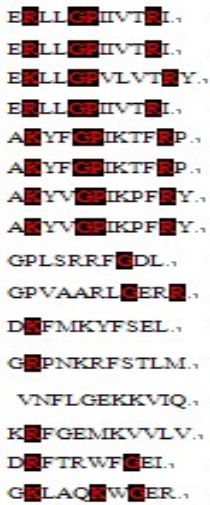 \\
\hline
\end{tabular}

stresses.

\section{MFS TRANSPORTERS AND STRESSES}

Multidrug transporters of major facilitator superfamily follow the same pattern to export the substrates from cell in exchange for pumping $\mathrm{H}^{+}$into the cell. However, there are some questions still to be obscure. For example, under what condition do the toxic compound export from the cell, and how do the transmembrane transporters change their conformation to antiport the substances at both sides of membranes. It is preliminarily considered ZIF1 probably transport the excess $\mathrm{Zn}$ harmful to cells to vacuole through trans-vacuole-membrane transport of Zn-ligand as a multidrug transporters of MFS in arabidopsis thaliana [14]. Thus, delicate mechanism of MFS transmembrane transporters remains further study. Many studies demonstrate that multidrug transporters of major facilitator superfamily play important roles in withstanding harmful stresses, for example, which are involved in the transport of suger [15-16], phosphates [17-18] and nitrate[19-20] in the identified members of MFS family of arabidopsis thaliana [8], and most of them are implicated in responding to

\section{A. MFS Multidrug Transporters and Pathogens}

Studies showed that some multidrug transporters are involved in the interaction between plants and pathogen. Plant pathogenic fungi utilize MFS multidrug transporter to export and deliver their own infectious agents to plants such as toxins, and play a critical role in toxicity during the process of infection. It is reported a gene ----TOXA, which carried two copies and encoded a HC-toxin efflux pump of the major facilitator superfamily [21]. This protein involves in the secretion of the host specific toxin cyclic tetrapeptide HC-toxin across the plasma membrane of the maize pathogen cochliobolus carbonum, and the HC-toxin function as histone deactetylases in many organisms. The deletion mutant of single copy of TOXA still could produce HC-toxin and be poisonous to maize; It is suggested the HC-toxin efflux pump became a pathogenic factor of $C$. carbonum encoded by TOXA through expelling the HC-toxin to maize. There was another best studied MFS multidrug transporter, CFP protein [22], which could produce polyketide cercosporin poisonous to soybean by a soybean pathogen Cercospora.kikuchii and contain 14 
transmembrane domains act as a pathogenic factor. Further studies showed that this protein leading to the disease of soybean,,which involved in the secretion of cercosporin from $C$. kikuchii to soybean, nevertheless, mutant $\triangle C F P$ reducing the toxicity to soybean with no cercosporin. $M g M f s 1$, a gene of major facilitator superfamily, was identified from the wheat pathogen fungal Mycosphaerella graminicola, the encoded protein contain 14 transmembrane spannersand which is high homology to the MFS drug: $\mathrm{H}^{+}$ transporters[23], It is suggested that this protein particaipated in the secretion of cercosporin and the inhibition of various drugs.A gene named tep /was found by Pieter, which shows high homology to the members of MFS transporters and encodes a transmemberane protein , conferring chlotamphenicol resistance in Sinorhizobium meliloti by expelling the antibiotic outside the bacteria[24]. The identification of tep 1 mutant found there is no feedback regulation of nodulation in $S$. meliloti, but the accumulation of the Nod factor precursor N-acetyl glucosamine in $S$. meliloti inhibits the expression of nod gene and reduces the nodule formation efficiency, It is suggested that Tep1 could transport the high concentration of $\mathrm{N}$-acetyl glucosamine in $S$. meliloti and lead to a reduction of nod gene expression in alfalfa plants.

There are other MFS transporters such as plant MFS drug efflux proteins can reduce the accumulation of toxins in body to defend the infection of bacteria pathogen through expelling the secondary metabolites produced by pathogen poisonous to plants. For example, a new defense-inducible gene $Z m-m f_{s} 1$ has been identified in the process of infection of pathogen, which encoded a MFS transporter with high homology to the bacterial multidrug efflux protein[12]. Its expression is induced by pathogen both in resistant and susceptible plants, reseach does further speculation that $Z m-m f s 1$ produced by infection of pathogen whose protein likely play a role in resisting the intrusion of pathogens by expelling the potential toxic compounds from plant pathogens. And the function of Zm-Mfs1 may cooperate with detoxification systems such as plant cytochrome P450 to reduce the loading of toxins in body[25].

\section{B. MFS Multidrug Transporters and Toxins}

MFS drug transporters with broader substrate specificity are able to transport kinds of toxic compounds mainly including endogenous and exogentic or nature and biosynthetic substrances. MFS drug transporters are involved in resistance to biotic stress by reducing the accumulation of toxic compounds in the cell [4, 26-27]. Research showed the multidrug resistance of bacteria poses a serious threat to the effects of the drugs. Overexpression of MFS drug transporters has been shown to protect the cell from toxic compounds by decreasing the accumulation of those toxins.

2,4-D is one of the synthetic auxin herbicides, which is extensively used owing to relatively modest toxicity and biodegradability in soil [28]. Research showed there were two genes of herbicide 2,4-D tolerance in saccharomyces cerevisiae, PDR5 and TPO1, respectively. PDR5 encoded a multidrug plasma membrane transporter of $\mathrm{ABC}$ family and TPO1 encoded a MFS drug: $\mathrm{H}^{+}$transporter $\mathrm{DHA}$, they transported a broad range of structurally distinct substrates from the cell [29-30]. The gene At5g13750 homogenous to the TPO1, screened with herbicide 2,4-D in Arabidopsis thaliana. The protein-structure prediction and analyses found that it belongs to the major facilitator superfamily and contains 12 transmembrane spanners locating in plasma membrane. The heterologous expression of this gene in $S$. cerevisiae found that the present of At5g13750 compensated for the functional deficiencies of resistance to 2,4-D owing to knockout of TPO1 gene in mutant $\triangle$ tpol. This transmembrane protein could expel 2,4-D under the energy by protein motive force to protect $S$. cerevisiae from 2,4-D by reducing the intracellular concentration of 2,4-D in $S$. cerevisiae [28].

\section{MFS Multidrug Transporters and Heavy Metal Stress}

The essential microelements in plant growth contain $\mathrm{Fe}$, $\mathrm{Mu}, \mathrm{Zn}, \mathrm{Cu}, \mathrm{B}, \mathrm{Mo}, \mathrm{Ni}$ and so on, but are also toxic to cells in excess. MFS drug efflux protein thus as E.coli TetB, which function as electrical neutral antiporter system to promote $\mathrm{H}^{+}$uptake and a divalent metal-tetracycline complex export [30-31].

The gene zifl (zinc-induced facilitator1) was obtained which located in the tonoplast and encoded a transmembrane transporter of major facilitator superfamily[14]. Futher investigation demonstrated the susceptible phenotype of zifl-1 mutant more serious than mtp1-1, and zifl-1 mtp1-1double mutants showed higher sensitivity than both of zifl-1 and mtp1-1 single mutant, illustrating ZIF1 might have been located in the tonoplast and independent of MTP1 as a transporter to participate in transporting $\mathrm{Zn}$ to the vacuole. The increased accumulation of $\mathrm{Zn}$ in shoots of arabidopsis thaliana zifl mutants might result from the function loss of ZIF1 in roots, leading to the decreased sequestration of vacuole to $\mathrm{Zn}$, and making $\mathrm{Zn}$ redistributed from roots to shoots. The expression of ZIF1p-GUS was strongest in the young tissues and mainly in the vasculature in older tissues. This result suggested that ZIF1 could transport $\mathrm{Zn}$ from roots to shoots by vasculature, and ZIF1 plays a key role in keeping the $\mathrm{Zn}$ homeostasis through transport the excess $\mathrm{Zn}$ to the vacuole as transmembrane transporter or the position with lower $\mathrm{Zn}$ concentration to reduce injury caused by the excess $\mathrm{Zn}$. Based on the high amino acid sequence similarity, they found some putative ZIF1 orthologs in other species. The study showed the expression of ZIF1 was strongly induced not only by Zn,but also by Mn to a lesser extent, however, not by $\mathrm{Fe} \cdot \mathrm{Cd}$ and $\mathrm{Cu}$, perhaps, this transporter participate in the translocation of various heavy metals under the induction of these heavy metals.

\section{MECHANISMS OF MFS MULTIDRUG TRANSPORTERS}

MFS multidrug transporters belong to sencondary transporter system, transporters of DHA and DHA2 subfamilies through pumping $\mathrm{H}^{+}$into the cell and exporting the toxic compounds outside or to the region of lower toxins concentration, making the concentration of toxin existence region decreased and protecting the cell from the damage of toxins[32-34]. The export of drugs and the uptake of $\mathrm{H}^{+}$ apparently require the help of additional auxiliary proteins, for example, the membrane fusion protein (MFP) and outer 
membrane factor (OMF) and so on.

It is found that N-terminal regions of MFS transporters are primarily involved in the proton transport, and $\mathrm{C}$ terminal regions are primarily involved in recognition and connection of substrates[9]. The formula: IS (integration score $)=\mathrm{CN}$ (co-evolutionary coupling score $) \times$ sequence conservation score was builded to identify the functionally important residues of multidrug transporters in MFS, the result found that the interation network of LacY and GlpT covered both of the 6-TMs, and the interation network of EmrD were found from only N-terminal symmetric half involved in the proton transport [35]. It is confirmed $\mathrm{N}$-terminal of transporters are involved in the proton transport, and C-terminal are involved in recognition and connection of substrates. It is presumed that both symmetric halves with different functions respectively possess their own specific functional cooperative residues. However how does the $\mathrm{N}$-terminal transport $\mathrm{H}^{+}$into the cell, and how does the C-terminal recognize and connect the substrates are waiting for further studies.

It is suggested that the relative importance of proton concentration gradient $(\Delta \mathrm{pH})$ and /or the membrane potential $(\Delta \Psi)$ depends on the condition of electrification of substrates. For example, MFS multidrug transporter MdfA supplies energy in different ways to the differently charged substates, MdfA energized by the $\Delta \mathrm{pH}$ and $\Delta \Psi$ while transporting neutral compounds, and energized only by the $\Delta \mathrm{pH}$ while transporting monocationic compound [36]. There are the same transport processes in LmrP and SMR transporters. Given multidrug transporters may seem promiscuous in their electrogenicity, they are strictly restricted to the transport stoichiometry. So far, there is no one trivalent cations that were found to be exported by any MFS transporters, since no transporters are able to import three protons while exporting substrates, so, the transport stoichiometry might be a key factor to decide which substrates are transported outside the cell.

MFS transporters have a broader substrate specificity, and there is how do these transporters transport different substrates. It is speculated that substrate selectivity of MFS transporters are not a "key-lock" static relationship, but a kinetic processes depending on the movement of transporters, connection of substrates to transporters and the affinity of substratesto bind the sites of transporters. Anyone of transporters possesses the specific ways to connect substrates [37]. Furthermore, since the uncertain conformation of transporters in each cycle, conformational change can be presumed by analyzing the formation and breaking of salt bridge in the process of substrate translocation. The MD simulations and biochemistry experiments of GlpT, OxlT and EmrD structure showed that antiporters with a single binding site medicate the substrate translocation by alterating access of rocker-switch mechanism[3].

\section{CONCLUSIONS}

MFS multidrug transporters are a class of important membrane transporters, which participate in facilitating the release of toxic metabolites and substances, maintaining the communication between cell and the environment, keeping the intracellular homeostasis and acting as a necessary element of energy generation and metabolic system, which has become a hot topic in the field of adversity biology and structural biology. With the rapid development of bioinformatics and molecular experimental techniques, function of MFS transporters could be identified at the levels of amino acids using mutants obtained by gene knockout, overexpression and heterologous expression. In recent years, the high resolution $3 \mathrm{D}$ crystal structures of members of major facilitator superfamily are showed with the development of electron crystal and 3D structures[6]. These structural characteristics provide some valuable information for detecting the protein function, meanwhile, can offer the help to completely understand the transport mechanism of MFS. For example, the conserved cores of evolutionarily coupled residues in functional restriction sites were effectively showed, and specific functional residues of MFS transporters were identified using integrative evolutionary analysis[35], these results provided the helpful information to identify functional amino acids of MFS transporters in the future.

Despite some progress has been made in studies of MFS transporters, however, the results are limited, some critical questions need to be resolved, such as detailed structure mechanism, patterns of binding the substrates, complex regulation mechanism of network consist of upstream and/or downstream genes, patterns of transmembrane transport and transport mechanism and so on. Moreover, there are few reports about the transport of secondary metabolites by MFS multidrug transports, and the regulation mechanism and the signal transduction pathways need the further studies. Most of the studies use the model plant Arabidopsis thaliana as materials, however, which are suitable for genomic research and may not be favorable as the secondary metabolite materials, so, other model plants suitable for the studies of secondary metabolites might supply the most important information for studying the transport mechanism of secondary metabolites in plants. The further studies of structure and transport mechanism can deepen the understanding of the important roles and delicate mechanism of transporters in the network of responding to stresses, and can decrease the damage of the tixic compounds thus as pesticide and heavy metal to plants, provide the fundamental theories and the actual using value for cultivating the excellent crops with high resistance to drugs and low accumulation of toxins combining genetic engineering technique with traditional breeding.

\section{ACKNOWLEDGMENT}

Hua Peng, Sufen Han and Mao Luo contributed equally to the review.

\section{REFERENCES}

[1] I. Paulsen. Multidrug efflux pump and resistance: regulation and evolution. Curr Opin Microbiol, 2003, 6(5): 446-451.

[2] I. Paulsen, M. Brown, R. Skurray. Proton-dependent multidrug efflux systems. Microbiol Rev, 1996, 60(4): 575-608.

[3] C. Law, P. Maloney C, D. Wang. Ins and outs of major facilitator superfamily antiporters. Annu Rev Microbio , 2008, 62: 289-305.

[4] S. Pao, I. Paulsen, M. Saier. Major facilitator superfamily. Microbiol Mol Biol Rev, 1998, 62:1-34. 
[5] M. Saier, J. Beatty, A. Goffeau, K. Harley, W. Heijne, S. Huang, D. Jack, P. Jähn, K. Lew, J. Liu, S. Pao, I. Paulsen, T. Tseng, P. Virk. The major facilitator superfamily. J Mol Microbiol Biotechnol, 1999, 1(2):257-79.

[6] Q. Ren, and I. Paulsen. Large-scale comparative genomic analyses of cytoplasmic membrane transport systems in prokaryotes. $\mathrm{J} \mathrm{Mol}$ Microbiol Biotechnol, 2007, 12(3-4): 165-179.

[7] A. Chang, R. Lin, W. Keith Studley, C. Tran, M. Saier. Phylogeny as a guide to structure and function of membrane transport proteins. Mol Membr Biol, 2004, 21(3):171-181.

[8] Q. Ren, and I. Paulsen. Comparative analyses of fundamental differences in membrane transport capabilities in prokaryotes and eukaryotes. PLoS Comput Biol, 2005, 1(3):e27.

[9] J. Griffith, M. Baker, D. Rouch, M. Page, R. Skurray, I. Paulsen, K. Chater, S. Baldwin,P. Henderson. Membrane transport proteins: implications of sequence comparisons. Curr Opin Cell Biol, 1992, 4(4): 684-695.

[10] M. Saier. Tracing pathways of transport protein evolution. Mol Microbiol, 2003, 48(5): 1145-1156.

[11] I.Sá-Correia, and S. Tenreiro. The multidrug resistance transporters of the major facilitator superfamily, 6 years after disclosure of Saccharomyces cerevisiae genome sequence. J Biotechnol, 2002, 98(2-3): 215-226.

[12] C. Simmons, M. Fridlender, P. Navarro, N. Yalpani. A maize defense-inducible gene is a major facilitator superfamily member related to bacterial multidrug resistance efflux antiporters. Plant $\mathrm{Mol}$ Biol, 2003, 52(2): 433-446.

[13] J. Ditty, and C. Harwood. Conserved cytoplasmic loops are important for both the transport and chemotaxis functions of PcaK, a protein from Pseudomonas putida with 12 membrane-spanning regions. $J$ Bacteriol, 1999, 181(16): 5068-5074.

[14] M. Haydon, and C. Cobbett. A novel major facilitator superfamily protein at the tonoplast influences zinc tolerance and accumulation in Arabidopisis. Plant Physiol, 2007, 143(4): 1705-1719.

[15] R. Lemoine. Sucrose transporters in plants: update on function and structure. Biochim Biophys Acta, 2000, 1465(1-2): 246-262.

[16] B. Quirino, W.Reiter, R. Amasino. One of two tandem Arabidopsis genes homologous to monosaccharide transporters is senescence-associated. Plant Mol Biol, 2001, 46(4): 447-457.

[17] U. Muchhal, J. Pardo, K. Raghothama. Phosphate transporters from the higher plant Arabidopsis thaliana. Proc Natl Acad Sci USA, 1996, 93(19): 10519-10523.

[18] F. Smith, P. Ealing, B. Dong, E. Delhaize. The cloning of two Arabidopsis genes belonging to a phosphate transporter family. Plant $J, 1997,11(1): 83-92$.

[19] L. Trueman, A. Richardson, B. Forde G. Molecular cloning of higher plant homologues of the high-affinity nitrate transporters of Clamydomonas reinhardtii and Aspergillus nidulans. Gene, 1996, 175(1-2): 223-231

[20] Y. Tsay, J. Schroeder, K. Feldmann, N. Crawford. The herbicide sensitivity gene Chll of Arabidopsis encodes a nitrate-inducible nitrate transporter. Cell, 1993, 72(5): 705-713.

[21] J. Pitkin, D. Panaccione, J. Walton. A putative cyclic peptide efflux pump encoded by the TOXA gene of the plant-pathogenic fungus Cochliobolus carbonum. Microbiology, 1996, 142( Pt 6): 1557-1565

[22] T. Callahan, M. Rose, M. Meade, M. Ehrenshaft, R. Upchurch. CFP, the putative cercosporin transporter of Cercospora kikuchii, is required for wild type cercosporin production, resistance, and virulence on soybean. Mol Plant Microbe Interact, 1999, 12(10): 901-910.
[23] R. Roohparvar, M. De Waard, G. Kema, L. Zwiers. MgMfs1, a major facilitator superfamily transporter from the fungal wheat pathogen Mycosphaerella graminicola, is a strong protectant against natural toxic compounds and fungicides. Fungal Genet Biol. 2007, 44(5):378-88.

[24] P. van Dillewijn, J. Sanjuán, J. Olivares, M. Soto. The tep1 gene of Sinorhizobium meliloti coding for a putative transmembrane efflux protein and $\mathrm{N}$-acetyl glucosamine affect nod gene expression and nodulation of alfalfa plants. BMC Microbiol. 2009, 9:17.

[25] F. Van Bambeke, E.Balzi, P. Tulkens. Antibiotic efflux pumps. Biochem Pharmacol, 2000, 60(4): 457-470.

[26] B. Bauer, H. Wolfger, K. Kuchler. Inventory and function of yeast ABC proteins: about sex, stress, pleiotropic drug and heavy metal resistance. Biochimica Biophysica Acta, 1999, 1461(2): 217-236.

[27] B. Nelissen, R. De Wachter, A. Goffeau. Classification of all putative permeases and other membrane plurispanners of the major facilitator superfamily encoded by the complete genome of Saccharomyces cerevisiae. FEMS Microbiol Rev, 1997, 21(2): 113-134.

[28] T. Cabrito, M. Teixeira, A. Duarte, P. Duque, I. Sá-Correia. Heterologous expression of a Tpol homolog from Arabidopsis thaliana confers resistance to the herbicide 2,4-D and other chemical stresses in yeast. Appl Microbiol Biotechnol, 2009, 84(5): 927-936.

[29] I. Sá-Correia, S. dos Santos, M. Teixeira, T. Cabrito, N. Mira. Drug:H antiporters in chemical stress response in yeast. Trends Microbiol, 2009, 17(1):22-31.

[30] M. Aldema, L. McMurry, A. Walmsley, S. Levy. Purification of the Tn10-specified tetracycline efflux antiporter TetA in a native state as a polyhistidine fusion protein. Mol Microbiol, 1996, 19(1): 187-195.

[31] Y. Someya, Y. Moriyama, M. Futai, T. Sawai, A. Yamaguchi. Reconstitution of the metal-tetracycline/H1 antiporter of Escherichia coli in proteoliposomes including F0F1-ATPase. FEBS Lett, 1995, 374(1):72-76.

[32] L. Grinius, and E. Goldberg. Bacterial multidrug resistance is due to a single membrane protein which functions as a drug pump. $J$ Biol Chem, 1994, 269(47):29998-30004.

[33] D. Ma, D. Cook, M. Alberti, N. Pon., H. Nikaido, J. Hearst. Genes acrA and acrB encode a stress-induced efflux system of Escherichia coli. Mol Microbiol, 1995, 16(1): 45-55.

[34] I. Paulsen, T. Littlejohn, P. Rådström, L.Sundström, O. Sköld, G. Swedberg, R. Skurray. The 3' conserved segment of integrons contains a gene associated with multidrug resistance to antiseptics and disinfectants. Antimicrob Agents Chemother, 1993, 37(4):761-768.

[35] J. Jeon, J. Yang, S. Kim. Integration of evolutionary features for the identification of functionally important residues in major facilitator superfamily transporters. PLoS Comput Biol, 2009, 5(10): e1000522

[36] A. Stapleton, and V. Walbot. Flavonoids can protect maize DNA from the induction of ultraviolet radiation damage. Plant Physiol, 1994, 105(3): 881-889.

[37] R. Ernst, P. Kueppers, J. Stindt, K. Kuchler, and L. Schmitt. Multidrug efflux pumps: substrate selection in ATP-binding cassette multidrug efflux pumps-first come, first served? FEBS $J, 2010$ 277(3): 540-549. 